\title{
Synthesis and characterization of a novel modified expandable graphite and its application in rigid polyurethane foams
}

\author{
Lin Liu ${ }^{1,2, a}$, Xiangjun Ma ${ }^{1, b}$, Rui Lv ${ }^{1, c}$, Zhengzhou Wang ${ }^{1,2 *, d}$
}

1 School of Materials Science and Engineering, Tongji University, Shanghai 201804, P.R. China

2 Key Laboratory of Advanced Civil Engineering Materials (Tongji University), Ministry of Education, Shanghai 201804, P.R. China

allsp@tongji.edu.cn, b1433019@tongji.edu.cn, ${ }^{\mathrm{c}} 1631394 @$ tongji.edu.cn, ${ }^{\mathrm{t}}$ zwang@tongji.edu.cn

Keywords: DOPO, modified expandable graphite, rigid polyurethane foam, flame retardation, mechanical properties

Abstract. In this work, an intermediate product DOPO-A was firstly synthesized through an addition reaction between triethoxyvinylsilane and 9,10-dihydro-9-oxa-10-phosphaphenanthrene-10-oxide (DOPO). Then DOPO-A was hydrolyzed to form DOPO-A' (silanol of DOPO-A) and the surface of expandable graphite (EG) was modified by DOPO-A' to prepare a novel modified expandable graphite (MEG). The effects of MEG on the flame-retardant and mechanical properties of rigid polyurethane (RPU) foams were investigated. It is found that MEG is more effective in improving the limiting oxygen index (LOI) of RPU foams than EG. The cone calorimeter studies indicate that MEG flame retarded RPU foams have lower peak heat release rate, total heat release, peak smoke production rate, and $\mathrm{CO}$ yield than those of the EG flame retarded RPU foams. Compressive strengths of the MEG flame retarded RPU foams have a great improvement compared with those of EG flame retarded RPU foams.

\section{Introduction}

Rigid polyurethane (RPU) foams are widely used in building external wall insulation, piping insulation, cold storage insulation and other fields because of their excellent thermal insulation performance [1]. But the poor flame resistance of polyurethane foams heavily impedes their practical applications [2-4]. As an inorganic intumescent flame retardant, expandable graphite (EG) can meet the requirements of RPU foams for flame retardation and environmental protection, and has attracted more attention these years [5-7]. However, there are some problems such as uneven distribution and the poor compatibility with the interface of RPU foams, which reduces the mechanical properties of RPU foams and limits the applications of EG $[8,9]$. So it is necessary to improve the interfacial adhesion between EG and RPU foams. One approach is to encapsulate the EG by some polymers. For example, Ye [10] reported that the EG particles were encapsulated with a layer of PMMA, and this method improved the dispersion of EG in PU matrix. Another alternative approach is to modify the surface of EG by introducing some functional groups. $\mathrm{Wu}$ [11] successfully prepared the silane functionalized $\mathrm{EG}$ by the 3-aminopropyltriethoxsilane (APTS)

9, 10-dihydro-9-oxa-10-phosphaphenanthrene-10-oxide (DOPO) and its derivatives are efficient phosphorus-containing flame retardants and have been widely researched in recent years [13, 14]. DOPO can release low molecular weight phosphorous substance which could scavenge the $\mathrm{H}$ - and $\mathrm{OH}$. radicals in the flame to hinder the spread of fire [15]. Due to the aromatic structures, DOPO-containing compounds have higher thermal and chemical stability than other linear low molecular weight phosphonates. In addition, silicone element has been applied in flame retardants to improve flammability of polymers [16,17]. In this paper, a silane coupling agent, i.e. triethoxyvinylsilane (A-151) was utilized to improve the compatibility between EG and foams matrix, while DOPO was used as phosphorus-containing flame retardant to improve the flame-retardant properties of RPU 
foams. To our best knowledge, there is little study of EG modified by a silane-DOPO composite (MEG) and applied it in the RPU foams.

In this work, the MEG was prepared by the modification of EG using the reaction product of DOPO and A-151, and it was characterized using Fourier transform infrared spectrometry (FTIR) and X-ray photoelectron spectroscopy (XPS). The effects of MEG on the flame-retardant, mechanical and thermal properties of RPU foams were investigated.

\section{Experimental}

\section{Materials}

Polyaryl polyisocyanate (PM-200) and polyether polyol mixture (RB2005-101) were supplied by Wanhua Chemical Group Co., Ltd., China. DOPO was purchased from Shouguang Weidong Chemical Co., Ltd., China. Triethoxyvinylsilane (A-151) was received from Nanjing Capatue Chemical Co., Ltd., China. Expandable graphite (EG), 80 meshes diameter, was obtained from Shijiazhuang ADT carbonic material factory, China. Azodiisobutyronitrile (AIBN), diethylene glycol dimethyl ether, acetic acid, cyclohexane and absolute ethyl alcohol were purchased from Sinopharm Chemical Reagent Co., Ltd., China.

\section{Preparation of modified expandable graphite (MEG)}

DOPO $(0.06 \mathrm{~mol})$ and diethylene glycol dimethyl ether $(120 \mathrm{ml})$ were charged into a four-necked round-bottomed flask equipped with a magnetic stirrer, a thermometer and a condensation reflux device in an oil bath at $80{ }^{\circ} \mathrm{C}$. The stirring speed was adjusted to $400 \mathrm{rpm}$ to make the DOPO dissolved completely. Then $20 \mathrm{ml}$ diethylene glycol dimethyl ether dissolved A-151 (0.05 mol) and AIBN $(0.001 \mathrm{~mol})$ were added through a constant pressure funnel, and the dropping-time was about 0.5 hour. The reaction was carried out at $80{ }^{\circ} \mathrm{C}$ for 24 hours to obtain DOPO-A. And then some acetic acid were added to regulate the $\mathrm{pH}$ of solution $(\mathrm{pH} 4-5)$ to make DOPO-A hydrolyze for half an hour to achieve DOPO-A'. Then 20g EG were charged into the solution, and the reaction was maintained for 6 hours. The modified expandable graphite (MEG) was filtered and washed several times with absolute ethyl alcohol, and finally dried in a vacuum oven. Fig. 1 shows the synthesis route of MEG.

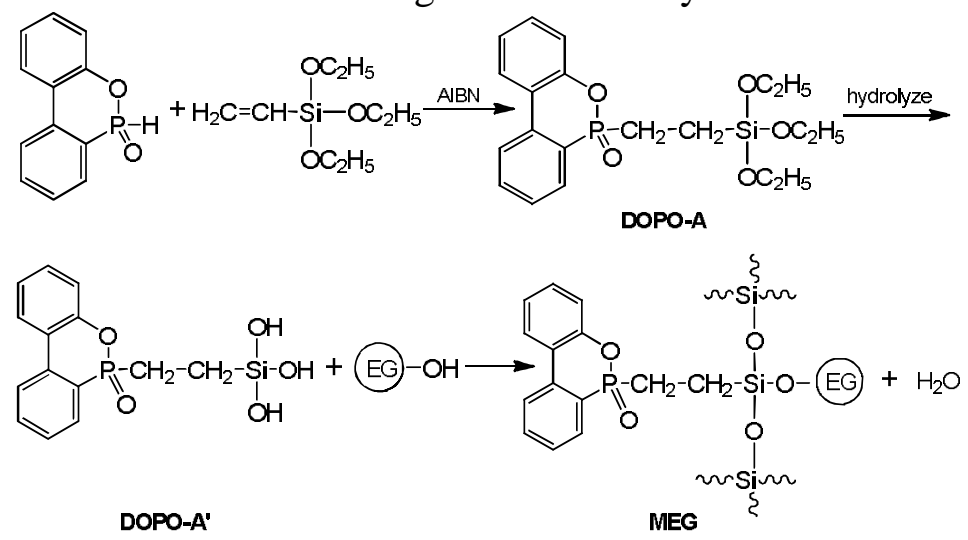

Fig. 1. Synthesis route of MEG

\section{Preparation of RPU foams}

A series of flame retarded RPU foams were prepared by the one-pot and free-rise method. The polyether polyol mixture (RB2005-101) was first premixed at room temperature with EG or MEG, and then PM-200 (the mass ratio of RB2005-101 and PM-200 was 1:1.5) was added into the above mixture and stirred at $1500 \mathrm{rpm}$ for $10 \mathrm{~s}$. Finally, the mixture was casted in an open mould $(20 \mathrm{~cm} \times 10$ $\mathrm{cm} \times 10 \mathrm{~cm}$ ) for foaming. The resulted RPU foams were placed in an oven for 24 hours at $60{ }^{\circ} \mathrm{C}$ to complete the polymerization reaction. Table 3 presents the formulations of pure RPU foam and flame retarded RPU foams obtained.

\section{Characterizations and tests}

The chemical structure of DOPO-A was determined by FTIR spectra and ${ }^{1} \mathrm{H}$ nuclear magnetic resonance $\left({ }^{1} \mathrm{H}\right.$ NMR) spectroscopy. FTIR spectra were obtained using an EQUNOX55 spectrometer 
(Bruker Corporation, Germany), and the samples were scanned at room temperature over a range of wavenumber from $600 \mathrm{~cm}^{-1}$ to $4000 \mathrm{~cm}^{-1}$. The ${ }^{1} \mathrm{H}$ NMR analysis was performed on an AVANCE III NMR spectrometer (Bruker Corporation, Switzerland) at $300 \mathrm{MHz}$, and the samples were dissolved in deuterated chloroform $\left(\mathrm{CDCl}_{3}\right)$ at a concentration of $1 \%(\mathrm{w} / \mathrm{v})$.

X-ray photoelectron spectra (XPS) was recorded using ESCALAB 250Xi (Thermo Electron Corporation, America), with AL-Ka excitation radiation at $15 \mathrm{kv}$, and the atmospheric pressure was maintained at about $1 \times 10^{-7} \mathrm{~Pa}$.

The thermal decomposition of the RPU foams was studied using a thermal analyzer (STD Q600, TA Instruments, America) and the temperature was scanned from room temperature to $900 \square$ at a heating rate of $10 \square / \mathrm{min}$ in nitrogen atmosphere.

Limiting oxygen index (LOI) tests were performed at room temperature using an oxygen index instrument (HC-2, Jiangning Analysis Instrument Co., Ltd. China). The size of the specimen was 100 $\mathrm{mm} \times 10 \mathrm{~mm} \times 10 \mathrm{~mm}$.

Cone calorimetry test was carried out using a FTT0007 cone calorimeter (Fire Testing Technology Limited, England). Each specimen, with the dimensions of $100 \mathrm{~mm} \times 100 \mathrm{~mm} \times 20 \mathrm{~mm}$, was wrapped in aluminium foil and exposed horizontally to an external heat flux of $35 \mathrm{kw} / \mathrm{m}^{2}$.

Density of the foams was measured according to GB-T-6343.The size of the specimen for the measurement was $50 \mathrm{~mm} \times 50 \mathrm{~mm} \times 50 \mathrm{~mm}$, and the average density was obtained from at least five repeat tests.

Compressive strength tests for the foams were performed using a universal mechanical tester (SANS7 CMT- 5105, Shenzhen SANS Testing Machine Co., Ltd. China). The size of the specimen was $20 \mathrm{~mm} \times 20 \mathrm{~mm} \times 20 \mathrm{~mm}$ and the crosshead speed was $2 \mathrm{~mm} / \mathrm{min}$.

\section{Results and discussion}

\section{Characterization of MEG}

\section{FTIR and ${ }^{1} H$ NMR}

The intermediate product DOPO-A obtained from DOPO and A-151 was filtered and washed several times with cyclohexane and dried in a vacuum oven for the characterization. The DOPO-A was determined by FTIR and ${ }^{1} \mathrm{H}$ NMR. Fig. 2 shows the FTIR spectra of DOPO and DOPO-A. The P-Ph stretching vibration is at $1477 \mathrm{~cm}^{-1}$, and the peak around $912 \mathrm{~cm}^{-1}$ is attributed to the $\mathrm{P}-\mathrm{O}-\mathrm{Ph}$ deformation vibration [18]. The peak at $1199 \mathrm{~cm}^{-1}$ belongs to the vibration of $-\mathrm{P}=\mathrm{O}$. The peak around $3060 \mathrm{~cm}^{-1}$ is assigned to the stretching vibration of Ar-H. The stretching vibration at $2438 \mathrm{~cm}^{-1}$ for $\mathrm{P}(\mathrm{O})-\mathrm{H}$ is observed in DOPO and disappears in DOPO-A [19]. This result speculates that the $\mathrm{P}(\mathrm{O})-\mathrm{H}$ bonds are exhausted in the reaction between DOPO and A-151.

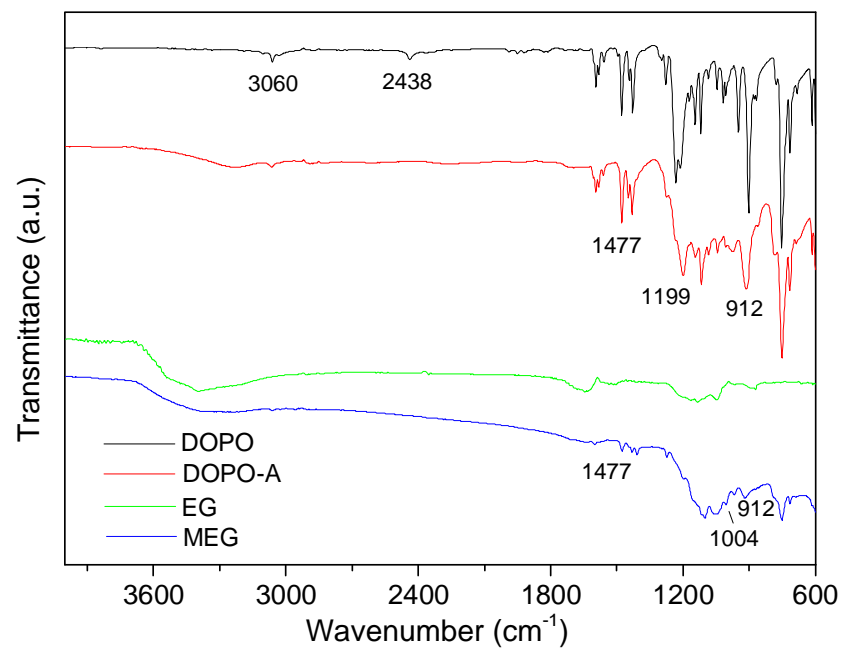

Fig. 2. FTIR spectra of DOPO, DOPO-A, EG and MEG

In order to confirm the reaction between DOPO and A-151, the structure of DOPO and DOPO-A were characterized by ${ }^{1} \mathrm{H}$ NMR. As shown in Fig. 3, the peak at $8.8 \mathrm{ppm}$ is associated with the P-H of 
DOPO [19], which disappears in the ${ }^{1} \mathrm{H}$ NMR spectra of DOPO-A, implying that the reaction happened between the $\mathrm{C}=\mathrm{C}$ of $\mathrm{A}-151$ and the $\mathrm{P}-\mathrm{H}$ of DOPO. Furthermore, some new peaks at $\sim 4.0$ ppm, $\sim 1.3 \mathrm{ppm}, \sim 2.0 \mathrm{ppm}$ and $\sim 0.8 \mathrm{ppm}$ are presented in Fig. 3, which are probably attributed to the proton of $-\mathrm{OCH}_{2} \mathrm{CH}_{3}$ (d) (e), $-\mathrm{P}-\mathrm{CH}_{2}$ (b) and $-\mathrm{CH}_{2}-\mathrm{Si}$ (c), respectively. The peaks at $7.1 \sim 8.2 \mathrm{ppm}$ are due to the $\mathrm{Ar}-\mathrm{H}$ (a) protons, and the peak at $\sim 1.4 \mathrm{ppm}$ is owed to the protons of cyclohexane.
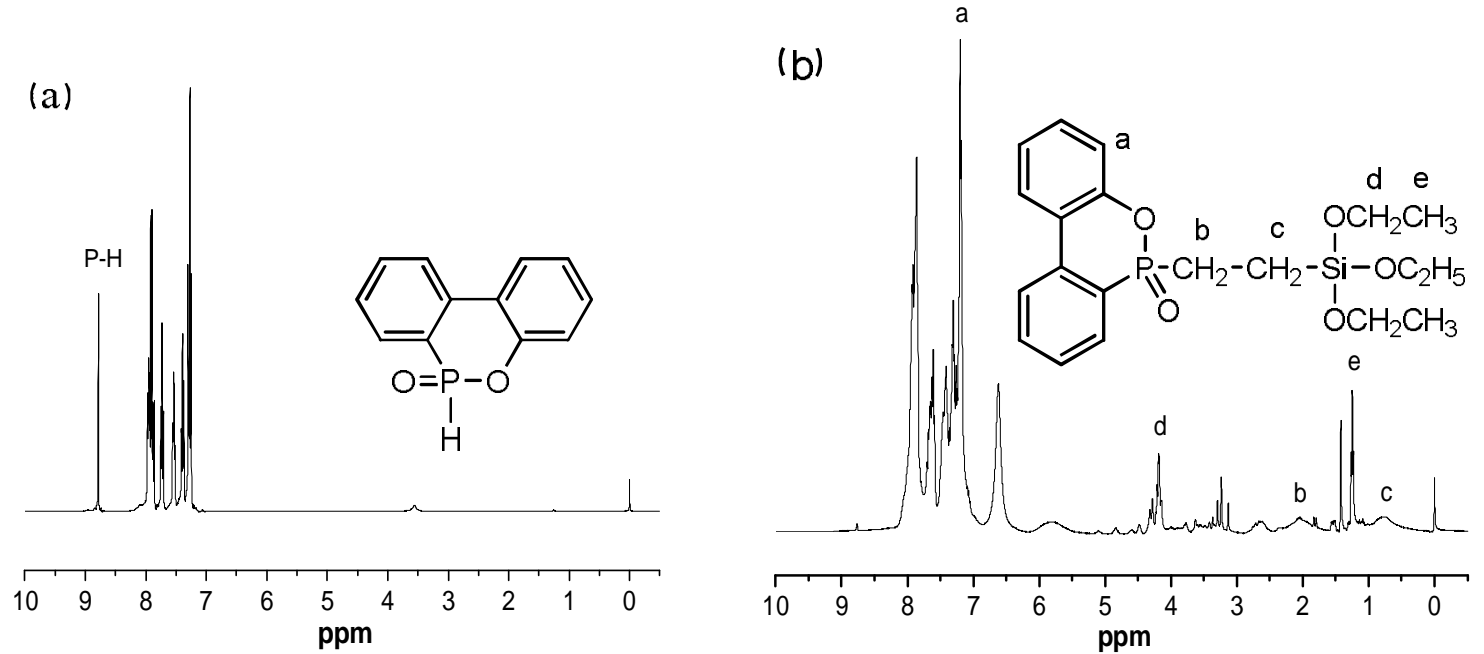

Fig. 3. ${ }^{1} \mathrm{H}$ NMR spectra of DOPO (a) and DOPO-A (b)

The FTIR spectra of EG and MEG are also displayed in Fig. 2 to verify the existence of DOPO-A' on the surface of EG. It can be seen that the characteristic peak of P-Ph stretching vibration of DOPO-A at $1477 \mathrm{~cm}^{-1}$ is clear in the spectra of MEG. The P-O-Ph deformation vibration peak at 912 $\mathrm{cm}^{-1}$ of DOPO-A appears in MEG too. In addition, the peak at $1004 \mathrm{~cm}^{-1}$ of Si-O-EG stretching vibration appears in MEG while does not appear in EG or DOPO-A [11], indicating that the reaction occurred between silanol (-Si-OH) and hydroxyl of EG. All these characteristic peaks does not appear in EG, implying that MEG has been successfully modified by DOPO-A'.

XPS analysis

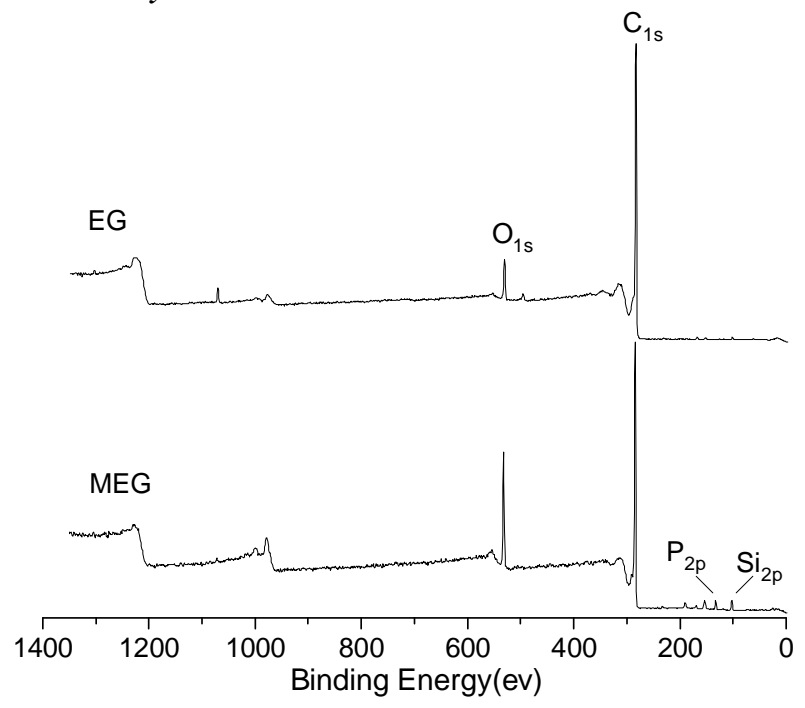

Fig. 4. XPS spectra of EG and MEG

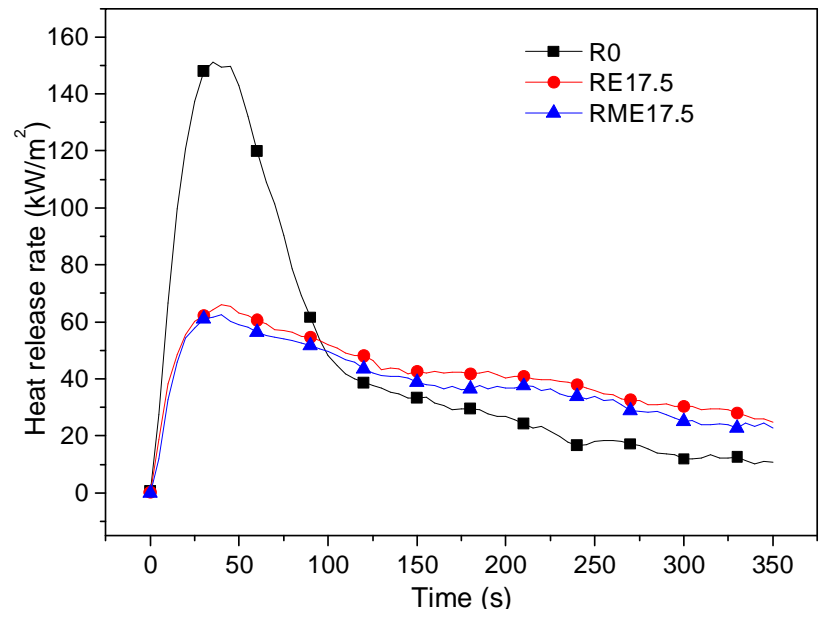

Fig. 5. HRR curve of R0, RE17.5 and RME17.5 XPS measurements provide further evidence of the reaction between the DOPO-A' and the EG. Fig. 4 displays the XPS spectra of EG and MEG. The $P_{2 p}$ peak does not include in EG while appears in MEG, and the intensity of $\mathrm{Si}_{2 \mathrm{p}}$ peak is obviously higher in MEG [20], which indicates that DOPO-A' has been attached to the surface of EG. Table 1 presents the relative element content of EG and MEG. The Si element content in EG is $0.84 \%$, that probably be attributed to the introduction of impurities when the raw materials were manufactured. The P and Si element content in MEG are 2.24\% and 3.16\%, respectively, that verifies the $\mathrm{P}$ and $\mathrm{Si}$ elements are contained in the surface of MEG. The $\mathrm{O}$ element content of MEG is higher than that of EG because of the introduction of oxygen-containing functional 
groups. The increase of $\mathrm{P}, \mathrm{Si}$ and $\mathrm{O}$ element content results in the decrease of $\mathrm{C}$ element content in MEG (77.9\%) compared with that of EG (92.16\%).

Table 1. Element content of EG and MEG

\begin{tabular}{lllll}
\hline Samples & $\mathrm{C}(\%)$ & $\mathrm{O}(\%)$ & $\mathrm{Si}(\%)$ & $\mathrm{P}(\%)$ \\
\hline EG & 92.16 & 5.74 & 0.84 & 0 \\
MEG & 77.9 & 15.14 & 3.16 & 2.24 \\
\hline
\end{tabular}

\section{Effect MEG on flame retardant properties of RPU foams}

\section{LOI analysis}

Table 3 reveals the effect of EG or MEG content on limiting oxygen index (LOI) values of RPU/EG foams (RPU foams filled with EG) or RPU/MEG foams (RPU foams filled with MEG). The LOI of neat RPU foam (R0) is only $18 \%$. LOI increases gradually with the increase of EG or MEG content, but MEG is more effective in improving LOI than EG at the same loading. For example, LOI value with the addition of $17.5 \mathrm{phr}$ MEG (RME17.5) increases to 30\% compared with $29 \%$ of $17.5 \mathrm{phr}$ EG (RE17.5). This phenomenon may be owed to the loading of phosphorus-silicon elements on the surface of MEG, which can play a synergistic flame retardant effect and improve the flame-retardant properties of RPU foams [21].

\section{Cone calorimeter analysis}

The cone calorimeter test (CCT) was also used to evaluate the flammability and smoke emission of RPU foams. Fig. 5 presents heat-release rate (HRR) curve of R0, RE17.5 and RME17.5. Some other CCT data are summarized in Table 2. The peak heat-release rate (PHRR) of R0 is $151.1 \mathrm{~kW} / \mathrm{m}^{2}$, and the PHRR of RE17.5 and RME17.5 are significantly reduced to $66.1 \mathrm{~kW} / \mathrm{m}^{2}$ and $62.5 \mathrm{~kW} / \mathrm{m}^{2}$, respectively, which implies that both EG and MEG are good PHRR reducers, and MEG is more effective than EG in decreasing PHRR of RPU foams. Moreover, the total heat release (THR) for RME17.5 is $13.56 \mathrm{MJ} / \mathrm{m}^{2}$ and decrease by $8.4 \%$ compared with the value of RE17.5 $\left(14.81 \mathrm{MJ} / \mathrm{m}^{2}\right)$ as shown in Table 2 . The above results are possibly because of the formation of good intumescent char from MEG, and there may be some synergistic flame retardant effect between the phosphorus and silicone containing DOPO-A' and EG [22].

The smoke emission behavior of materials is very important for the personal safety during the fire. It can be seen from Table 2 that the peak smoke production rate (PSPR) of RME17.5 is $0.034 \mathrm{~m}^{2} / \mathrm{s}$, which exhibits a $24 \%$ reduction compared to the value of RE17.5 $\left(0.045 \mathrm{~m}^{2} / \mathrm{s}\right)$ and a $52 \%$ reduction compared to the value of R0 $\left(0.071 \mathrm{~m}^{2} / \mathrm{s}\right)$. Compared with the R0, the total smoke release (TSR) values of RE17.5 and RME17.5 all decrease greatly. The $\mathrm{CO}$ yield and $\mathrm{CO}_{2}$ yield of RME17.5 decrease by $45.3 \%$ and $6.1 \%$ compared with that of R0, and decrease by $3.6 \%$ and $3.1 \%$ compared with that of RE17.5 respectively. These data indicate that MEG has a better performance in the suppression of smoke and toxic gas emission than EG.

Table 2. CCT data for R0, RE17.5 and RME17.5

\begin{tabular}{ccccccc}
\hline Samples. & $\begin{array}{c}\text { PHRR } \\
\left(\mathrm{kW} / \mathrm{m}^{2}\right)\end{array}$ & $\begin{array}{c}\text { THR } \\
\left(\mathrm{MJ} / \mathrm{m}^{2}\right)\end{array}$ & $\begin{array}{c}\text { PSPR } \\
\left(\mathrm{m}^{2} / \mathrm{s}\right)\end{array}$ & $\begin{array}{c}\mathrm{CO} \text { yield } \\
(\mathrm{kg} / \mathrm{kg})\end{array}$ & $\begin{array}{c}\mathrm{CO}_{2} \text { yield } \\
(\mathrm{kg} / \mathrm{kg})\end{array}$ & $\begin{array}{c}\mathrm{TSR} \\
\left(\mathrm{m}^{2} / \mathrm{m}^{2}\right)\end{array}$ \\
\hline R0 & 151.1 & 16.02 & 0.071 & 0.148 & 1.32 & 343.7 \\
RE17.5 & 66.1 & 14.81 & 0.045 & 0.084 & 1.28 & 97.6 \\
RME17.5 & 62.5 & 13.56 & 0.034 & 0.081 & 1.24 & 96.7 \\
\hline
\end{tabular}

\section{Effect MEG on mechanical properties of RPU foams}

The influence of EG or MEG content on density and compressive strength of RPU foams are shown in Table 3. It can be observed that the density of RPU foams increases while increasing the EG or MEG content up to $20 \%$. Compressive strength depends greatly on the apparent density of foams [19], and thus the compressive strength increases with the increase of the density. Compared with RPU/EG foams, RPU/MEG foams have a higher density and compressive strength at the same content. Furthermore, $\sigma$ (compressive strength/density) is applied for distinguishing the compressive strength 
on the same density basis [23]. Fig. 6 shows the effect of EG or MEG content on $\sigma$ values of RPU foams. The $\sigma$ values of flame retardant RPU foams decrease while increasing the EG or MEG content. Compared with the neat RPU foam (R0), the $\sigma$ of RPU foam containing 10 phr EG (RE10) decreases by $14.2 \%$. RPU/MEG foams have an improvement in $\sigma$ values compared with RPU/EG foams. For example, the $\sigma$ of RPU foam containing $10 \mathrm{phr}$ MEG (RME10) is $4.8 \%$ higher than that of the RE10. The increased compressive strengths of RPU/MEG foams may be attributed to the improvement of compatibility between MEG and RPU foams matrix.

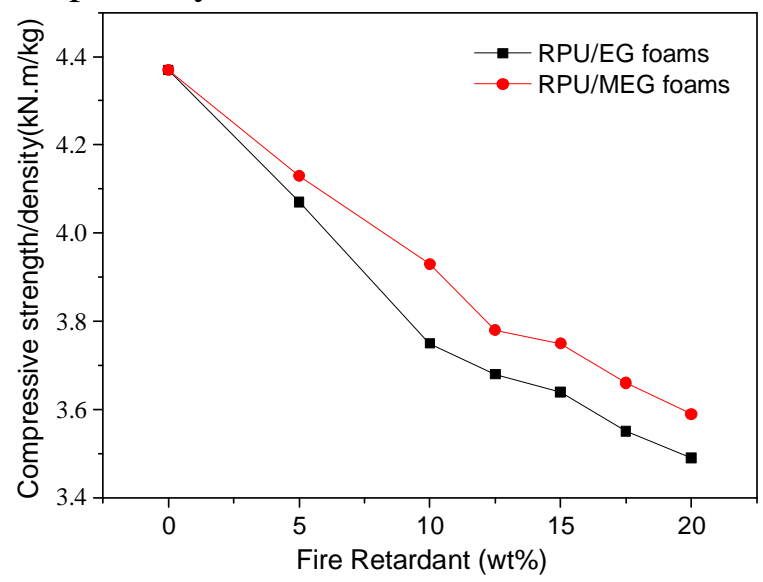

Fig. 6. Effect of EG or MEG content on $\sigma$ of RPU foams

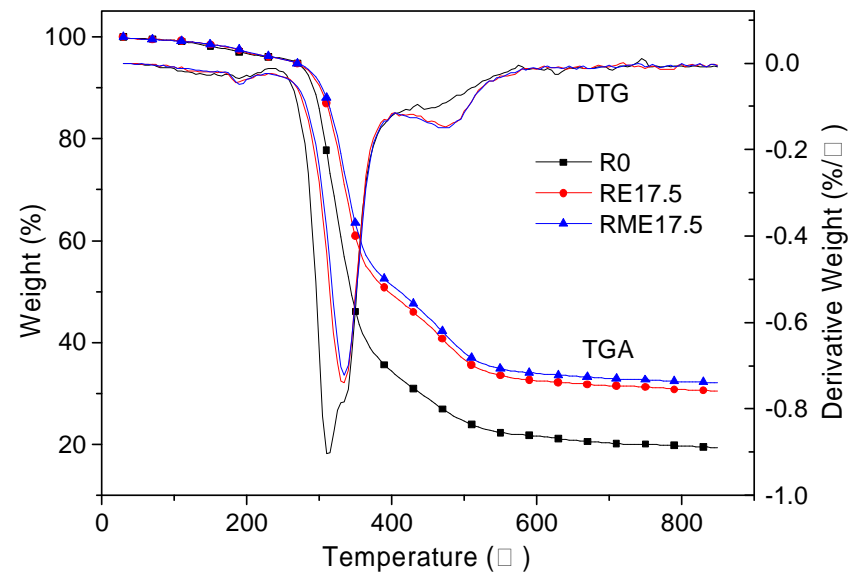

Fig. 7. TGA and DTG cures of R0, RE17.5 and RME17.5

Table 3. Flame retardant and mechanical properties of flame retardant RPU foams

\begin{tabular}{lccccccc}
\hline $\begin{array}{l}\text { Sample } \\
\text { code }\end{array}$ & $\begin{array}{c}\text { RPU } \\
(\mathrm{phr})\end{array}$ & $\begin{array}{c}\text { EG } \\
(\mathrm{phr})\end{array}$ & $\begin{array}{c}\text { MEG } \\
(\mathrm{phr})\end{array}$ & $\begin{array}{c}\text { LOI } \\
(\%)\end{array}$ & $\begin{array}{c}\text { Density } \\
\left(\mathrm{kg} \cdot \mathrm{m}^{-3}\right)\end{array}$ & $\begin{array}{c}\text { Compressive } \\
\text { strength }(\mathrm{MPa})\end{array}$ & $\begin{array}{c}\sigma \\
\left(\mathrm{kN} \cdot \mathrm{m} \cdot \mathrm{kg}^{-1}\right)\end{array}$ \\
\hline R0 & 100 & 0 & 0 & 18 & 41.2 & 0.180 & 4.37 \\
RE5 & 100 & 5 & 0 & 22 & 42.5 & 0.173 & 4.07 \\
RE10 & 100 & 10 & 0 & 24 & 50.6 & 0.190 & 3.75 \\
RE12.5 & 100 & 12.5 & 0 & 26 & 56.5 & 0.208 & 3.68 \\
RE15 & 100 & 15 & 0 & 27 & 60.4 & 0.220 & 3.64 \\
RE17.5 & 100 & 17.5 & 0 & 29 & 64.7 & 0.230 & 3.55 \\
RE20 & 100 & 20 & 0 & 32 & 67.5 & 0.236 & 3.49 \\
RME5 & 100 & 0 & 5 & 23 & 43.8 & 0.181 & 4.13 \\
RME10 & 100 & 0 & 10 & 25 & 51.1 & 0.201 & 3.93 \\
RME12.5 & 100 & 0 & 12.5 & 27 & 57.4 & 0.217 & 3.78 \\
RME15 & 100 & 0 & 15 & 28 & 61.5 & 0.231 & 3.75 \\
RME17.5 & 100 & 0 & 17.5 & 30 & 65.3 & 0.239 & 3.66 \\
RME20 & 100 & 0 & 20 & 33 & 68.3 & 0.245 & 3.59 \\
\hline
\end{tabular}

\section{Thermal decomposition of MEG flame retarded RPU foams}

Fig. 7 presents the thermogravimetric analysis (TGA) and DTG curves of R0, RE17.5 and RME17.5. In Fig. 7, the char yield at $800 \square$ of RME17.5 is $32.4 \mathrm{wt} \%$, and increases by $5.2 \%$ in comparison with that of RE17.5 (30.8 $\mathrm{wt} \%$ ), indicating that MEG can enhance the thermal stability of RPU foams compared with EG.

\section{Conclusions}

Expandable graphite (EG) was successfully modified by the reaction product between 9,10-dihydro-9-oxa-10-phosphaphenanthrene-10-oxide (DOPO) and triethoxyvinylsilane (A-151), and the modified EG (MEG) was confirmed by FTIR and XPS. The effect of EG or MEG on flame-retardant, mechanical and thermal properties of rigid polyurethane (RPU) foams was evaluated. It is found that the LOI values of the RPU foam containing MEG are all higher than the values of the RPU foam containing EG at the same loading. The cone calorimeter studies indicate that MEG flame retarded RPU foams have lower PSPR, PHRR, $\mathrm{CO}$ yield, $\mathrm{CO}_{2}$ yield, THR and TSR compared with 
that of the RPU foams with EG. Moreover, the thermal stability of the RPU/MEG foams is higher than that of the RPU/EG foams.

\section{Acknowledgements}

This work was financially supported by the National Natural Science Foundation of China (No. U1205114).

\section{References}

[1] Y. H. Hu,Y. Gao,D. N. Wang, et al. J. Appl. Polym. Sci., 2002, 84(3), 591-597

[2] M. L. Hobbs, K. L. Erickson, T. Y. Chu, Polym. Degrad. Stab., 2000, 69(1), 47-66

[3] A. Kausar, M. Siddiq, Int. J. Polym. Anal. Charact., 2016, 21(5), 436-446

[4] A. Kausar, Int. J. Polym. Anal. Charact., 2016, 21(4), 275-285

[5] X. C. Bian, J. H. Tang, Z. M. Li, et al. J. Appl. Polym. Sci., 2007, 104(5), 3347-3355

[6] S. Duquesne, M. L. Bras, S. Bourbigot, et al. Fire. Mater., 2003, 27(3), 103-117

[7] Z. Wang, E. Han, W. Ke, Corros. Sci., 2007, 49(5), 2237-2253

[8] M. Modesti, A. Lorenzetti, F. Simioni, et al. Polym. Degrad. Stab., 2002, 77(2), 195-202

[9] Y. Liu, J. He, R. Yang, Ind. Eng. Chem. Res., 2015, 54(22), 5876-5884

[10]L. Ye, X. Y. Meng, X. Ji, et al. Polym. Degrad. Stab., 2009, 94(6), 971-979

[11]T. C. Wu, K. C. Tsai, M. C. Lu, et al. J. Compos. Mater., 2012, 46(12), 1483-1496

[12]C. F. Kuan, W. H. Yen, C. H. Chen, et al. Polym. Degrad. Stab., 2008, 93(7), 1357-1363

[13]K. A. Salmeia, S. Gaan, Polym. Degrad. Stab., 2015, 113, 119-134

[14]X. Xu, Y. Wu, C. Wang, Des. Monomers. Polym., 2015, 18(7), 690-697

[15]A. Schäfer, S. Seibold, W. Lohstroh, et al. J. Appl. Polym. Sci., 2007, 105(2), 685-696

[16]C. L. Chiang, C. C. M. Ma, Polym. Degrad. Stab., 2004, 83(2), 207-214

[17]A. Cireli, N. Onar, M. F. Ebeoglugil, et al. J. Appl. Polym. Sci., 2007, 105(6), 3748-3756

[18]M. Zhang, Z. Luo, J. Zhang, et al. Polym. Degrad. Stab., 2015, 120, 427-434

[19]P. Wang, F. Yang, L. Li, et al. Polym. Degrad. Stab., 2016, 129, 156-167

[20]C. H. Chen, W. H. Yen, H. C. Kuan, et al. Polym. Compos., 2010, 31(1), 18-24

[21] Y. L. Liu, C. I. Chou, Polym. Degrad. Stab., 2005, 90(3), 515-522

[22]Y. L. Liu, Y. C. Chiu, C.S. Wu, J. Appl. Polym. Sci., 2003, 87(3), 404-411

[23]M. Thirumal, D. Khastgir, G. B. Nando, et al. Polym. Degrad. Stab., 2010, 95(6), 1138-1145 